\title{
MicroRNAs for the Prediction of Early Response to Sorafenib Treatment in Human Hepatocellular Carcinoma
}

\author{
Naoshi Nishida Tadaaki Arizumi Satoru Hagiwara Hiroshi Ida \\ Toshiharu Sakurai Masatoshi Kudo \\ Department of Gastroenterology and Hepatology, Kindai University Faculty of Medicine, \\ Osaka, Japan
}

\section{Key Words}

Hepatocellular carcinoma - microRNA - Molecular targeted therapy · Biomarkers · Treatment

\begin{abstract}
Background: Several studies suggest the role of circulating microRNAs (miRNAs) as biomarkers of hepatocellular carcinoma (HCC). However, the serum miRNA profile associated with the response to sorafenib remains to be elucidated. The aim of this study was to clarify the specific miRNAs in serum that could predict the early response of HCC to sorafenib treatment. Summary: Analyzing the sera from 16 HCC patients, we selected five miRNAs that showed differences in serum levels between patients with and without tumor responses among 179 known secretory miRNAs by using locked nucleic acid probe-based quantitative PCR. Through further analysis using a validation cohort that included $53 \mathrm{HCC}$ patients who underwent sorafenib treatment and 8 healthy control subjects, we found that miR-181a-5p and miR-339$5 p$ showed significant differences in serum levels among patients with partial response (PR), stable disease (SD), and progressive disease (PD), where PR patients showed the highest and PD the lowest levels. We also analyzed the factors associated with disease control (DC; PR or SD) 3 months after the initiation of sorafenib treatment; patients with DC showed a significantly higher level of serum miR-181a-5p than non-DC patients or healthy control subjects ( $p=0.0349$ and 0.0180 for DC vs. non-DC and control vs. non-DC by Tukey-Kramer test, respectively). We further conducted multivariate analysis among HCC patients with Barcelona Clinic Liver Cancer stage $C$ using extrahepatic metastasis, serum decarboxyprothrombin, and miR-181a-5p levels as covariables; serum miR-181a-5p was the only independent factor for achieving DC ( $p=0.0092$, odds ratio 0.139 , and $95 \%$ confidence interval $0.011-0.658)$. In addition, miR-181a-5 $p$ level was also the only independent factor affecting overall survival $(p=$ 0.0194 , hazard ratio 0.267 , and 95\% confidence interval 0.070-0.818). Key Messages: A high serum level of miR-181a-5p before treatment is associated with DC after the initiation of sorafenib.




\section{Liver Cancer}

\section{Introduction}

Hepatocellular carcinoma (HCC) is one of the leading causes of cancer death worldwide [1]. Despite recent advancements in the diagnosis and treatment of HCC, the prognosis of the patients at an advanced stage, where curative treatments are not applicable, is still unsatisfactory.

Noncurative treatment of HCC mainly consists of transcatheter-based treatment and molecular targeting treatment [2]; to date, sorafenib is the only agent that has improved the time-to-progression and overall survival (OS) in advanced stages of HCC with vascular invasion and extrahepatic spread [3]. According to the Barcelona Clinic Liver Cancer (BCLC) algorithm that refers to disease staging and treatment allocation, sorafenib is proposed as a first-line treatment for cases with BCLC stage C, where cancer has spread into blood vessels or extrahepatic tissues [4]. However, a considerable number of HCC patients with BCLC stage $\mathrm{C}$ are refractory to sorafenib; some of these patients may have a survival benefit from transcatheter-based treatment if intrahepatic tumor could be considered as a main prognostic factor for survival [5]. On the other hand, a subset of HCC patients with BCLC stage B, where transcatheter chemoembolization (TACE) is recommended, might be refractory to TACE but respond to sorafenib [6]. From this point of view, it is important to develop a biomarker to narrow down the subgroup of the patients who have a survival benefit from sorafenib treatment.

Recently, a number of studies have reported that microRNAs (miRNAs) could be key players in the pathogenesis of liver cancer [7]. Given that a single miRNA targets multiple mRNAs, the alteration of multiple pathways attributed to the miRNA profile may act in concert and affect the sensitivity of HCC cells to sorafenib [8-10]. To date, several reports have suggested that miRNAs could influence the cellular response to drugs, and a profile of the miRNAs in peripheral blood could be biomarkers for the drug response of HCC [11-13]. However, the performance of miRNAs as biomarkers for the response to sorafenib treatment remains to be elucidated.

Moreover, several issues need to be addressed for the quantification of serum miRNAs [14]. For example, the low concentration of miRNAs in peripheral blood could give rise to unreliable quantification, and nonspecific miRNAs derived from apoptotic bodies due to the injury of liver tissue and blood cells could act as noise in the measurement of target miRNAs. In addition, a reference for the quantification of miRNAs in peripheral blood has not yet been established. In this study, we attempted to address these important issues using locked nucleic acid (LNA) probes for quantitative PCRs (qPCRs). LNA is a modified RNA nucleotide, and qPCR using LNA probes provides enhanced sensitivity and specificity for quantification [15]. In this study, we specifically focused on the 179 known secretory miRNAs present in peripheral blood, and performed quantification using several reference miRNAs that are abundant in serum as a control of quantification. These procedures allowed us to perform stable quantification and eliminate the background noise of miRNAs in serum. Using screening and validation cohorts, we examined the secretory miRNAs in peripheral blood that could help identify the subclass of HCC patients who would likely respond to sorafenib treatment.

\section{Materials and Methods}

Patients and Screening of Serum miRNAs Associated with Tumor Response to Sorafenib Therapy

For the screening of miRNAs that could predict the initial response to sorafenib, we selected 8 HCC patients who showed partial response (PR) to sorafenib treatment at 3 months after the initiation of the treatment as well as 8 patients with progressive disease (PD) at 1 month after treatment initiation (screening cohort). The sera were collected before the initiation of sorafenib. The tumor response was evaluated 


\section{Liver Cancer}

according to the criteria outlined in the modified Response Evaluation Criteria in Solid Tumors (mRECIST) [16]. The characteristics of the screening cohort that consisted of the PR and PD patients are shown in online supplementary table 1 (for all online suppl. material, see www.karger.com/doi/10.1159/000449475). Using the serum from the screening cohort that was collected before the initiation of sorafenib, we selected the miRNAs that showed differences in serum levels between the PR and PD cases.

In the next step, we selected 53 consecutive patients who underwent sorafenib treatment for more than 1 month between July 2009 and May 2013 as a validation cohort. Among the 53 patients, 12 received sorafenib treatment as an initial therapy for HCC. On the other hand, 41 patients underwent several treatments before sorafenib, and the last treatment before sorafenib was as follows: 26 patients received TACE, 7 underwent hepatic arterial infusion chemotherapy, 5 received radiofrequency ablation, one underwentsurgical resection and 2 received other molecular targeting agent.

The collection of serum was performed within 1 week prior to the initiation of treatment, and serum was stored at $-80^{\circ} \mathrm{C}$ until the measurement of miRNA. The characteristics of the validation cohort are listed in table 1. Briefly, the median age of the patients was 74 years of age (67.5-79.5), with 45 males and 8 females. Thirteen were positive for hepatitis surface antigen (HBsAg), 22 were positive for hepatitis $\mathrm{C}$ virus antibody, and 18 were negative for both. Eight patients were classified as Barcelona Clinic or Liver Cancer (BCLC) stage A, 15 were stage B, and 30 were stage $C$. Twenty-three patients showed extrahepatic metastasis and 11 had vascular invasion. The median duration of sorafenib treatment was 99 days (24-567 days). Fifteen patients discontinued sorafenib administration before the 3rd month of treatment due to the diagnosis of PD or noncompliance with treatment. We also measured the serum miRNA levels from 8 healthy volunteers ranging in age from 23 to 77 years old. Informed consent was obtained from each patient, and the study protocol conforms to the ethical guidelines of the 1975 Declaration of Helsinki. This study was approved by the institution's research committee.

Follow-Up and Assessment of Response

After the initiation of sorafenib, the response to the therapy was assessed at 1 and 3 months; complete response (CR), PR, stable disease (SD), and PD were determined based on the radiological findings obtained from contrast-enhanced computed tomography that were reviewed by 2 independent radiologists in a nonblinded fashion. Extrahepatic metastasis was also evaluated as required using chest X-ray, bone scintigraphy, or fluorodeoxyglucose positron emission tomography [6]. We also defined the disease control rate (DCR) as the total number of the patients with CR, PR, and SD divided by the number of all patients, and the patients were classified under disease control (DC) or not based on the contrast-enhanced computed tomography findings 3 months after the initiation of sorafenib [3]. Blood chemical tests, and serum $\alpha$-fetoprotein and decarboxyprothrombin (DCP) levels were also measured monthly.

\section{Extraction of Total RNA from Serum and cDNA Synthesis}

Total RNA including miRNA was isolated from $250 \mu \mathrm{l}$ of serum using the miRNeasy mini-kit according to the manufacturer's protocol (Qiagen, Hilden, Germany); $1 \mu \mathrm{g}$ of carrier MS2 RNA was added to each serum sample (F. Hoffmann-La Roche, Ltd, Basel, Switzerland). We also used a set of synthetic RNA spike-ins (RNA spike-in kit; UniRT) for quality control, such as monitoring RNA isolation efficiency, interplate calibration, cDNA synthesis, and qPCR (Exiqon, Vedbaek, Denmark). RNA was eluted into $50 \mu \mathrm{l}$ of DNase/RNase-free water. For the reverse transcription, $2 \mu \mathrm{l}$ of template total RNA solution was added to $2 \mu \mathrm{l}$ of $5 \times$ reaction buffer, $4.5 \mu \mathrm{l}$ of nuclease-free water, $1 \mu \mathrm{l}$ of enzyme mix, and $0.5 \mu \mathrm{l}$ of enzyme mix synthetic RNA spike-ins, according to the manufacture's protocol (Universal cDNA Synthesis Kit II; Exiqon).

\section{Quantification of Serum miRNA Levels}

For the screening of miRNAs that predict an early response to sorafenib treatment and reliable detection of low amounts of serum miRNA, we used a serum/plasma focus miRNA PCR panel containing 179 miRNA primer sets focused on secreted serum/plasma relevant human miRNAs, and ExiLENT SYBR Green master mix (Exiqon, Vedbaek, Denmark). For the stability measurements of low level of miRNAs, an LNA probe, a novel type of nucleic acid analog that could distinguish a 1-bp mismatch, was used to obtain enhanced sensitivity and specificity of qPCR. The qPCR was performed using the StepOne real-time detection system (Applied Biosystems, Foster City, Calif., USA). The expression level of five reference miRNAs abundantly detected in serum (miR-95-5p, miR-103a-3p, miR-191-5p, miR-423-3p, and miR-425-5p) was measured, and their mean value was applied as an internal control for quantification $[17,18]$. We applied the comparative cycle threshold method for relative quantification of miRNAs within the input according to the manufac- 
Nishida et al.: MicroRNAs for the Prediction of Early Response to Sorafenib Treatment in Human Hepatocellular Carcinoma

Table 1. Clinical characteristics of the validation cohort

\begin{tabular}{|c|c|}
\hline Clinical factors & Characteristics of the patients ${ }^{1}$ \\
\hline Age, years & $74(67.5$ to 79.5$)$ \\
\hline \multicolumn{2}{|l|}{ Sex } \\
\hline Male & 45 \\
\hline Female & 8 \\
\hline \multicolumn{2}{|l|}{ Etiology } \\
\hline HBV & 13 \\
\hline $\mathrm{HCV}$ & 22 \\
\hline NBNC & 18 \\
\hline Maximum tumor size, $\mathrm{cm}$ & $3.7(2.1$ to 7.25$)$ \\
\hline \multicolumn{2}{|l|}{ Number of tumors } \\
\hline$\leq 5$ & 25 \\
\hline$\geq 6$ & 28 \\
\hline \multicolumn{2}{|l|}{ BCLC stage } \\
\hline A & 8 \\
\hline $\mathrm{B}$ & 15 \\
\hline $\mathrm{C}$ & 30 \\
\hline \multicolumn{2}{|l|}{ Extrahepatic metastasis } \\
\hline Without & 30 \\
\hline With & 23 \\
\hline \multicolumn{2}{|l|}{ Portal vein thrombosis } \\
\hline Without & 42 \\
\hline With & 11 \\
\hline Serum AFP level before treatment, ng/ml & $70(10$ to 4,846$)$ \\
\hline Serum DCP level before treatment, mAU/ml & $1,185(68.5$ to 7,865$)$ \\
\hline \multicolumn{2}{|l|}{ Initial dose of sorafenib } \\
\hline $200 \mathrm{mg}$ & 1 \\
\hline $400 \mathrm{mg}$ & 16 \\
\hline $800 \mathrm{mg}$ & 36 \\
\hline Duration of sorafenib treatment, days & 99 (56 to 233.5) \\
\hline \multicolumn{2}{|l|}{ Response to sorafenib } \\
\hline$\Delta \mathrm{AFP}$ at 1 month after the initiation ${ }^{2}$ & $0.03(-0.27$ to 0.57$)$ \\
\hline$\Delta \mathrm{DCP}$ at 1 month after the initiation ${ }^{3}$ & $1.80(0.34$ to 3.31$)$ \\
\hline \multicolumn{2}{|l|}{ At 1 month after the initiation } \\
\hline PR & 11 \\
\hline SD & 22 \\
\hline $\mathrm{PD}^{4}$ & 20 \\
\hline \multicolumn{2}{|l|}{ At 3 months after the initiation } \\
\hline PR & 7 \\
\hline SD & 9 \\
\hline $\mathrm{PD}^{5}$ & 22 \\
\hline DCR at 3 months after the initiation ${ }^{6}$ & $16 / 53(30.2 \%)$ \\
\hline
\end{tabular}

AFP $=\alpha$-Fetoprotein. ${ }^{1}$ Median value (25th to 75 th percentiles) for contentious variables, and number of cases for categorical variables are shown as characteristics of the patients. ${ }^{2}$ Differences of serum AFP level between before and at 1 month after the initiation of sorafenib; the $\triangle \mathrm{AFP}$ is calculated as (AFP level after the treatment - baseline AFP level) divided by baseline AFP level. A minus score indicates a decrease in APF compared with a baseline value. ${ }^{3}$ Differences of serum DCP level between before and at 1 month after the initiation of sorafenib; the $\triangle \mathrm{DCP}$ is calculated as (DCP level after the treatment - baseline DCP level) divided by baseline DCP level. A minus score indicates a decrease in DCP compared with a baseline value. ${ }^{4}$ Response at 1 month after the initiation of sorafenib was not determined in one patients. ${ }^{5}$ Sorafenib treatment was stopped before the 3rd month in 15 patients because of the disease progression. These patients were not evaluated for response at 3 months after the initiation. ${ }^{6}$ DCR was calculated as total number of the patients with PR and SD divided by total number of all patients. 
Nishida et al.: MicroRNAs for the Prediction of Early Response to Sorafenib Treatment in Human Hepatocellular Carcinoma

Table 2. Candidates of serum miRNAs for the prediction of early response to sorafenib

\begin{tabular}{|c|c|c|c|c|c|c|}
\hline $\begin{array}{l}\text { Candidate } \\
\text { miRNA }^{1}\end{array}$ & $\begin{array}{l}\text { Mean } \\
\Delta \mathrm{Cq}\end{array}$ & $\begin{array}{l}\text { PR group, median } \\
\Delta \mathrm{Cq} \text { (distribution) }\end{array}$ & $\begin{array}{l}\text { PD group, median } \\
\Delta \mathrm{Cq} \text { (distribution) }\end{array}$ & $\begin{array}{l}\mathrm{p} \text { value by } \\
\text { Student's } \\
\text { t test }\end{array}$ & $\begin{array}{l}\mathrm{p} \text { value by } \\
\text { Mann- } \\
\text { Whitney U } \\
\text { test }\end{array}$ & $\begin{array}{l}\text { miRNA levels in } \\
\text { PR group } \\
\text { vs. PD group }\end{array}$ \\
\hline hsa-miR-136-5p & 6.707 & $5.572(4.954-7.162)$ & 7.957 (7.146-7.996) & 0.0046 & 0.0275 & increased \\
\hline hsa-miR-17-5p & 5.361 & $5.630(5.016-8.622)$ & $4.919(3.842-5.048)$ & 0.0144 & 0.0023 & decreased \\
\hline hsa-miR-181a-5p & 2.555 & $2.098(1.720-3.388)$ & $2.972(2.112-3.554)$ & 0.0238 & 0.0357 & increased \\
\hline hsa-miR-182-5p & 7.455 & $7.648(7.272-9.466)$ & $6.918(6.046-8.044)$ & 0.0275 & 0.0618 & decreased \\
\hline hsa-miR-33a-5p & 5.520 & $4.987(4.066-5.744)$ & $5.976(4.818-8.374)$ & 0.0342 & 0.0357 & increased \\
\hline hsa-miR-335-5p & 6.684 & $6.279(4.578-7.31)$ & $6.903(5.784-8.916)$ & 0.0423 & 0.0587 & increased \\
\hline hsa-miR-339-5p & 5.958 & $5.804(4.568-6.414)$ & $6.254(5.348-7.368)$ & 0.0428 & 0.0587 & increased \\
\hline hsa-miR-148b-3p & 2.068 & $1.950(0.628-2.270)$ & $2.474(1.574-3.118)$ & 0.0443 & 0.0587 & increased \\
\hline
\end{tabular}

${ }^{1}$ miRNAs that showed a difference of serum levels between PR and PD groups with $\mathrm{p}<0.05$ by Student's $\mathrm{t}$ test. miRNAs with $\mathrm{p}<0.05$ by Mann-Whitney $\mathrm{U}$ test, and mean $\Delta \mathrm{Cq}<6$ are shown in bold.

turer's instruction. We performed duplicate measurements per experiment, and the mean number of cycles required for the fluorescent signal to reach the threshold (quantification cycle; $\mathrm{Cq}$ ) was determined for each sample. The $\mathrm{Cq}$ was determined automatically by the StepOne real-time detection system (Applied Biosystems). The difference of $\mathrm{Cq}$ between the target miRNA and a mean of the five reference miRNAs ( $\Delta \mathrm{Cq})$ was considered as a relative value of serum miRNA level for each sample [18]. Specificity of amplification was confirmed using melting curve analysis for each amplification (online suppl. fig. 1). Since serum miRNAs derived from blood cells should affect the measurement of miRNA from HCC cells, the serum level of three miRNAs abundantly present in blood cells (miR-16, miR-451, and miR-23a) was also examined to monitor hemolysis [18]. For the validation of miRNAs that are associated with early response to sorafenib, we also applied LNA miRNA primer-based qPCR.

\section{Statistics}

For comparing contentious variables, the Wilcoxon rank-sum test, Kruskal-Wallis test and Student's t test, or one-way factorial analysis of variance (ANOVA) was applied. For multiple comparisons, the TukeyKramer test or Wilcoxon rank-sum test with Bonferroni correction was applied. For categorical comparisons of clinical data, the $\chi^{2}$ test or Fisher's exact test was used. For the interassay variation, Pearson's correlation test was applied; the percentage coefficient of variation $(\% \mathrm{CV})$ was calculated. For the survival analysis, we applied Kaplan-Meier analysis and calculated a p value using the log-rank test. Variables with a $\mathrm{p}$ value of $<0.05$ on univariate analysis were further analyzed by the Cox proportional-hazards regression model to determine the independent determinants of outcome variables. All $p$ values were two-sided, and $p<0.05$ was considered statistically significant. All statistical analyses were calculated using the JMP version 4.05J software (SAS Institute Inc., Cary, N.C., USA).

\section{Results}

\section{Screening of Serum miRNAs Associated with Early Response to Sorafenib}

Initially, we conducted a screening of miRNAs associated with early response to sorafenib among 179 secreted miRNAs in serum. For this purpose, we compared serum miRNA levels between 8 HCC patients who showed PR for 3 months (PR group) and 8 HCC patients who were diagnosed as PD 1 month after initiation of treatment (PD group) (online suppl. fig. 2). Eight miRNAs showed significant differences in serum levels between the PR and PD groups with p values of $<0.05$ by Student's t test (table 2). Among them, we further selected 5 miRNAs, miR-17-5p, miR-181a-5p, miR-33a-5p, miR-339-5p, and miR-148b-3p, as candidates for validation as these miRNAs showed differences in serum levels even with the nonparametric method ( $p<0.0587$ by Wilcoxon rank-sum test) and were present at a high enough concentration in serum to allow for quantification $(\Delta \mathrm{Cq}<6)$ (table 2). Of them, 4 showed a decrease 
in $\Delta$ Cq in the PR group compared with that of the PD group, and 1 miRNA showed an increased $\Delta \mathrm{Cq}$ in the PR group.

Initial Response to Sorafenib among HCC Patients in the Validation Cohort

Among the 53 patients in the validation cohort, no patients were diagnosed as CR at either 1 or 3 months after the initiation of treatment. Eleven (11/53, 20.8\%), 22 (22/53, $41.5 \%)$, and $20(20 / 53,37.7 \%)$ patients comprised the PR, SD, and PD groups at 1 month, respectively. Of them, 5 and 10 patients who were classified as SD and PD, respectively, 1 month after the initiation of sorafenib, discontinued treatment due to tumor progression. The response at 3 months was evaluated in 38 patients; 7 (7/38, 18.4\%), 9 (9/38, 23.7\%), and 22 $(22 / 38,57.9 \%)$ patients were diagnosed as PR, SD, and PD, respectively. No patients in the PR group at the first month of the treatment were diagnosed as CR afterwards. Thus, the DCR at 3 months, calculated as the number of patients with PR and SD for 3 months divided by the total number of patients who received sorafenib, was $30.2 \%(16 / 53)$.

\section{Validation of Serum miRNAs That Predict Early Response to Sorafenib}

We confirmed the association of serum miRNA levels with tumor response 1 and 3 months after the initiation of sorafenib for five different miRNAs selected from the validation cohort. Of them, miR-181a-5p and miR-339-5p showed significant differences in serum levels 1 month after the initiation of sorafenib among the PR, SD, and PD groups by both ANOVA and nonparametric Kruskal-Wallis tests $(\mathrm{p}=0.0223$ and 0.0256 by ANOVA and Kruskal-Wallis tests for miR-181a-5p, respectively; $p=0.0244$ and 0.0133 by ANOVA and Kruskal-Wallis tests for miR-339a-5p, respectively, fig. 1a, b). These miRNA levels also showed significant differences 3 months after treatment initiation among the sera from the three groups $(\mathrm{p}=$ 0.0333 and 0.0271 by ANOVA and Kruskal-Wallis tests for miR-181a-5p, respectively; $p=$ 0.0323 and 0.0290 by ANOVA and Kruskal-Wallis tests for miR-339a-5p, respectively, fig. 1c, d). Interestingly, there was a clear trend between tumor response and the $\Delta \mathrm{Cq}$ of miRNAs; in patients with PR it is the smallest, and in those with PD it is the largest (fig. 1).

Using healthy subjects as a control, we compared the serum miRNA levels between patients with and without DC 3 months after treatment. Although the $\Delta$ Cq of miR-339-5p is larger in patients without DC (non-DC) than those with DC by nonparametric analyses ( $\mathrm{p}=$ 0.0341 among 3 groups by the Kruskal-Wallis tests, and $p=0.0143$ between DC and non-DC by Wilcoxon rank-sum test with Bonferroni correction; fig. 2a), a significant difference was not detected using ANOVA ( $\mathrm{p}=0.0742)$. On the other hand, non-DC patients showed significantly larger $\Delta \mathrm{Cq}$ of miR-181a-5p than DC patients as well as control subjects in both parametric and nonparametric analyses $(\mathrm{p}=0.0048$ by ANOVA, $\mathrm{p}=0.0349$ and 0.0180 for DC vs. non-DC and control vs. non-DC by Tukey-Kramer test, respectively; $\mathrm{p}=0.0032$ by KruskalWallis tests, $\mathrm{p}=0.0150$ and $\mathrm{p}=0.0038$ for DC vs. non-DC and control vs. non-DC by Wilcoxon rank-sum test with Bonferroni correction, respectively; fig. 2b).

\section{Interassay Variation for Quantification of Serum miRNAs}

Using sera from 19 HCC patients, we evaluated interassay variation for miR-181a-5p and miR-338-5p. For this propose, we compared the $\Delta$ Cq based on the mean value of five reference miRNAs (shown in Materials and Methods) with those based on the mean of two reference miRNAs (miR-95-5p, miR-423-3p). The $\Delta$ Cq from two assays was more correlated in miR$181 \mathrm{a}-5 \mathrm{p}$ than in miR-338-5p $\left(\mathrm{R}^{2}=0.7804, \mathrm{p}<0.0001\right.$ for miR-181a-5p, and $\mathrm{R}^{2}=0.418, \mathrm{p}=$ 0.0068 for miR-339-5p by Pearson's correlation coefficient; fig. 3 ). In addition, the interassay $\% \mathrm{CV}$ is smaller in miR-181a-5p than in miR-338-5p (\%CV $=16.6$ and $28.6 \%$ for miR-181a-5p and miR-338-5p, respectively), suggesting that miR-181a-5p is more suitable for the evaluation of serum levels than miR-338-5p in a clinical setting. 


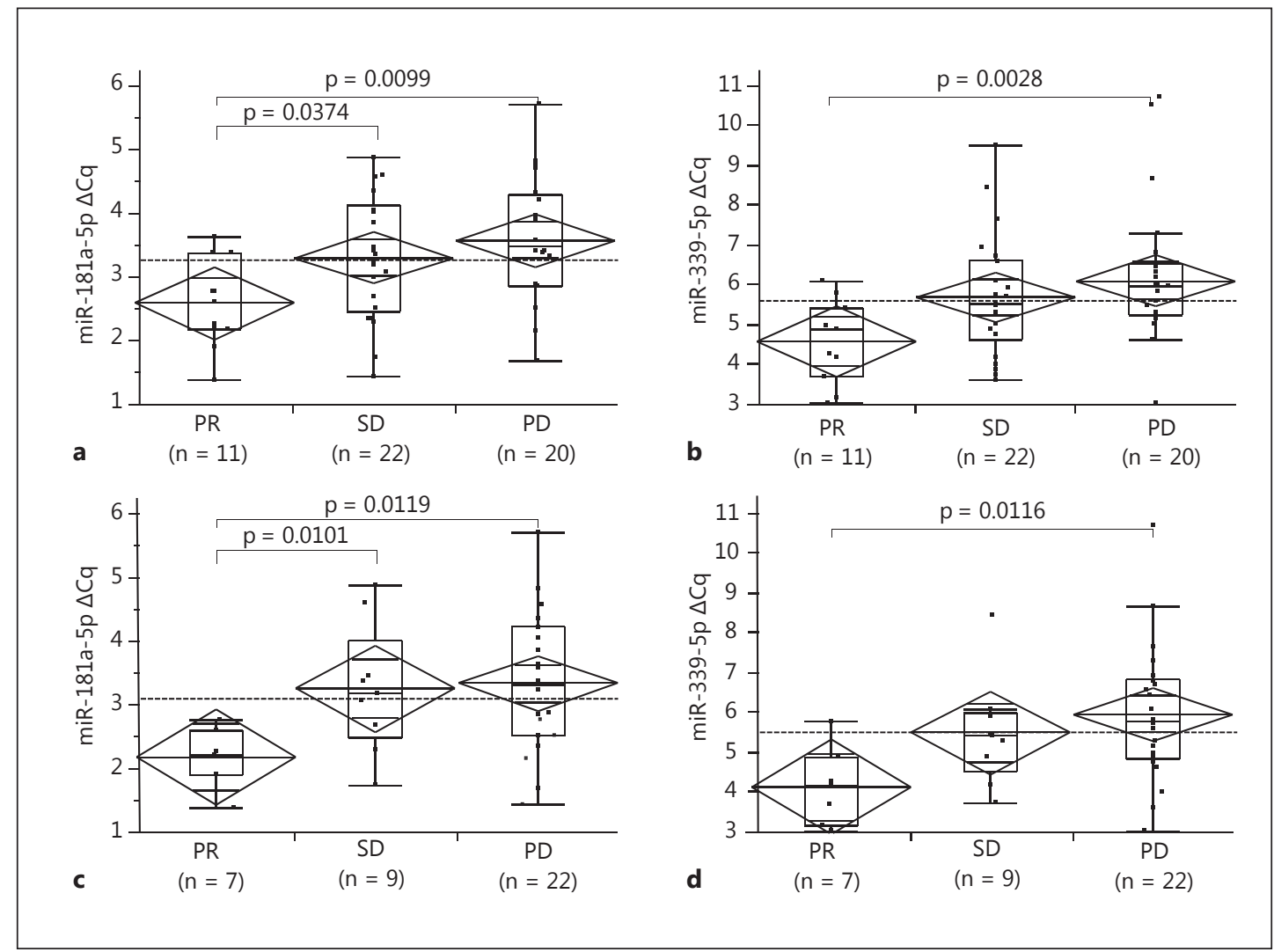

Fig. 1. Association between $\Delta \mathrm{Cq}$ of serum miRNAs and tumor response after the initiation of sorafenib. Diamond and lines in the diamond indicate the means and 95\% CIs of each subgroup; boxes and whiskers denote 75 and 95\% distributions, and the lines in the boxes show median values, respectively. Dashed lines in the each panel represent the mean value of all patients. p values between each group shown in the panels were calculated using Wilcoxon rank-sum test with Bonferroni correction. a $\Delta$ Cq of serum miR-181a-5p among the patients who showed PR, SD, and PD at 1 month after the initiation of sorafenib. The difference was significant between 3 groups by both ANOVA and Kruskal-Wallis tests $[p=0.0223, F(2,52)=4.12$ by ANOVA and $p=0.0256$ by Kruskal-Wallis tests, respectively]. $\mathbf{b} \Delta$ Cq of serum miR-339-5p among the patients who showed PR, SD, and PD at 1 month after the initiation of sorafenib. The difference was significant between 3 groups by both ANOVA and Kruskal-Wallis tests $[\mathrm{p}=0.0244, \mathrm{~F}(2,52)=4.00$ by ANOVA and $\mathrm{p}=0.0133$ by Kruskal-Wallis tests]. c $\Delta$ Cq of serum miR-181a-5p among the patients who showed PR, SD, and PD at 3 months after the initiation of sorafenib. The difference was significant between 3 groups by both ANOVA and Kruskal-Wallis tests $[p=0.0333, F(2,37)=3.76$ by ANOVA and $p=0.0271$ by Kruskal-Wallis tests]. $\mathbf{d} \Delta$ Cq of serum miR-339-5p among the patients who showed PR, SD, and PD at 3 months after the initiation of sorafenib. The difference was significant between 3 groups by both ANOVA and Kruskal-Wallis tests [p = $0.0323, \mathrm{~F}(2,37)=3.79$ by ANOVA and $\mathrm{p}=0.0290$ by Kruskal-Wallis tests].

\section{Clinical Factors That Predict DC for Sorafenib Treatment}

Before treatment, we studied clinical factors that were associated with DC. For this purpose, we categorized contentious variables of age, maximum tumor size, and number of tumor into two groups using their median values shown in table 1. Among the variables examined, BCLC stage, extrahepatic metastasis, DCP level before treatment, and the $\triangle \mathrm{Cq}$ of miR-181a-5p and miR-338-5p were significantly associated with DC at 3 months after sorafenib treatment $(\mathrm{p}=0.0093$ and 0.0173 for BCLC stage and extrahepatic metastasis by Pearson's $\chi^{2}$ test, respectively; $\mathrm{p}=0.0135,0.0146$, and 0.0139 for DCP level, and $\Delta$ Cq of miR- 

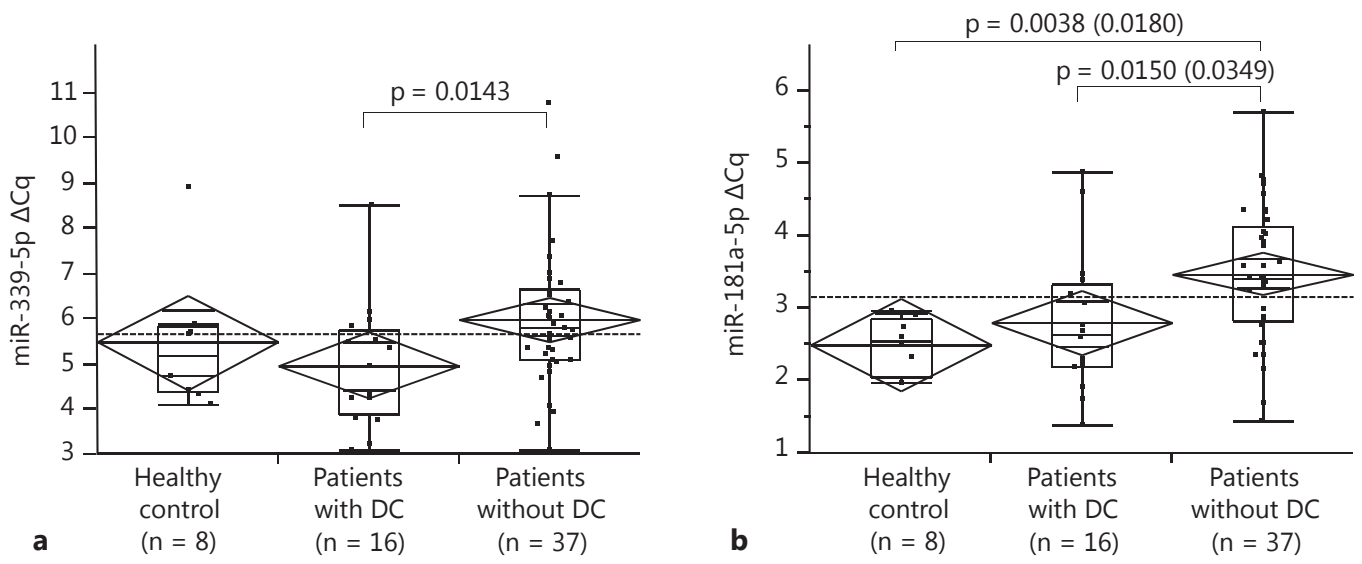

Fig. 2. Association between $\triangle \mathrm{Cq}$ of serum miRNAs and $\mathrm{DC}$ at 3 months after the initiation of sorafenib. Patients with DC were defined as patients with PR or SD for 3 months. Diamond and lines in the diamond indicate the means and 95\% CIs of each subgroup; boxes and whiskers denote 75 and 95\% distributions, and the lines in the boxes show median values, respectively. Dashed lines in each panel represent the mean value of all patients. p values between each group shown in the panels were calculated using Wilcoxon rank-sum test with Bonferroni correction. a $\Delta$ Cq of serum miR-339-5p among the healthy control, patients with and without DC at 3 months after the initiation of sorafenib. $p=0.0742, F(2,60)=2.72$ by ANOVA and $p=0.0341$ by Kruskal-Wallis tests, respectively. $\mathbf{b} \Delta$ Cq of serum miR-181a-5p among the healthy control and patients with and without DC at 3 months after the initiation of sorafenib. $p=0.0048, F(2,60)=5.87$ by ANOVA and $p=$ 0.0032 by Kruskal-Wallis tests. In addition to the $\mathrm{p}$ values calculated using Wilcoxon rank-sum test with Bonferroni correction ( $p=0.0150$ and 0.0038 for DC vs. non-DC and control vs. non-DC, respectively), the $p$ values by post hoc Tukey-Kramer test are also shown in parentheses ( $p=0.0349$ and 0.0180 for DC vs. non-DC and control vs. non-DC, respectively).

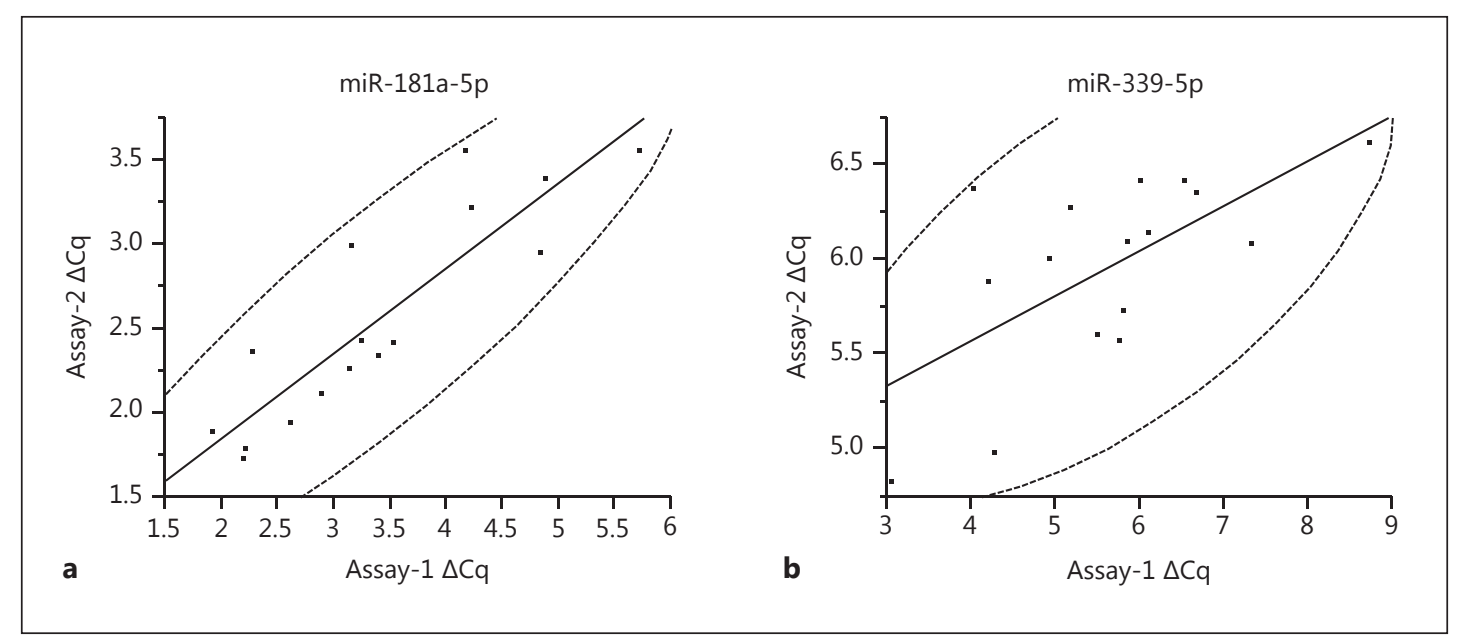

Fig. 3. Interassay variation for quantification of serum miRNAs. The $\Delta$ Cq values based on the mean value of 5 kinds of reference miRNAs (assay-1) and those based on the mean of 2 kinds of reference miRNAs (assay-2) were compared. The dashed curves represent $95 \%$ confidence ellipse area. a Interassay variation for $\Delta \mathrm{Cq}$ of serum miR-181a-5p. $\mathrm{R}^{2}=0.418, \mathrm{p}=0.0068$ by Pearson's correlation coefficient. $\mathbf{b}$ Interassay variation for $\Delta$ Cq of serum miR-339-5p. $\mathrm{R}^{2}=0.7804, \mathrm{p}<0.0001$ by Pearson's correlation coefficient. 
Nishida et al.: MicroRNAs for the Prediction of Early Response to Sorafenib Treatment in Human Hepatocellular Carcinoma

Table 3. Associations between characteristics before treatment and disease control after the initiation of sorafenib

\begin{tabular}{|c|c|c|c|c|c|}
\hline \multirow{2}{*}{$\begin{array}{l}\text { Characteristics before } \\
\text { treatment }\end{array}$} & \multirow[t]{2}{*}{ Patients with $\mathrm{DC}^{2}$} & \multirow[t]{2}{*}{ Patients without $\mathrm{DC}^{2}$} & \multirow{2}{*}{$\begin{array}{l}\text { Univariate } \\
\text { p value }^{3}\end{array}$} & \multicolumn{2}{|c|}{ Multivariate ${ }^{4}$} \\
\hline & & & & $\mathrm{p}$ value & OR $(95 \% \mathrm{CI})$ \\
\hline \multicolumn{6}{|l|}{$\mathrm{Age}^{1}$} \\
\hline$\leq 73$ years & 9 & 16 & 0.3839 & - & - \\
\hline$\geq 74$ years & 7 & 21 & & & \\
\hline \multicolumn{6}{|l|}{ Sex } \\
\hline Male & 13 & 32 & 0.6249 & - & - \\
\hline Female & 3 & 5 & & & \\
\hline \multicolumn{6}{|l|}{ Virus } \\
\hline HBV & 5 & 8 & 0.3039 & - & - \\
\hline $\mathrm{HCV}$ & 8 & 14 & & & \\
\hline NBNC & 3 & 15 & & & \\
\hline \multicolumn{6}{|l|}{$\operatorname{Size}^{1}$} \\
\hline$<3.6 \mathrm{~cm}$ & 7 & 19 & 0.6133 & - & - \\
\hline$>3.7 \mathrm{~cm}$ & 9 & 18 & & & \\
\hline \multicolumn{6}{|l|}{ Number of tumors ${ }^{1}$} \\
\hline$<5$ & 10 & 15 & 0.1415 & - & - \\
\hline$>6$ & 6 & 22 & & & \\
\hline BCLC stage & & & 0.0093 & 0.0475 & \\
\hline A & 4 & 4 & & & \\
\hline B & 8 & 7 & & & \\
\hline $\mathrm{C}$ & 4 & 26 & & & \\
\hline BCLC-B/A & & & & 0.7230 & $0.71(0.10-4.68)^{5}$ \\
\hline BCLC-C/A & & & & 0.0430 & $0.14(0.02-0.94)$ \\
\hline BCLC-C/B & & & & 0.0387 & $0.20(0.04-0.93)$ \\
\hline \multicolumn{6}{|l|}{ Extrahepatic metastasis } \\
\hline Without & 13 & 17 & 0.0173 & - & - \\
\hline With & 3 & 20 & & & \\
\hline \multicolumn{6}{|l|}{ Portal vein thrombosis } \\
\hline Without & 14 & 28 & 0.3298 & - & - \\
\hline With & 2 & 9 & & & \\
\hline $\begin{array}{l}\text { AFP level before } \\
\text { treatment, ng/ml }\end{array}$ & $158(16-1,527.25)$ & $65(7-5,367.5)$ & 0.8691 & - & \\
\hline $\begin{array}{l}\text { DCP level before } \\
\text { treatment, } \mathrm{mAU} / \mathrm{ml}\end{array}$ & $142.5(27.25-1,785.25)$ & $3,431(178.5-17,700)$ & 0.0135 & 0.9092 & $1(1-1)$ \\
\hline$\Delta \mathrm{Cq}$ of serum miR-181a-5p & $2.65(2.20-3.35)$ & $3.42(2.82-4.14)$ & 0.0146 & 0.2864 & $0.63(0.26-1.47)$ \\
\hline$\Delta \mathrm{Cq}$ of serum miR-339-5p & $4.92(3.86-5.73)$ & $5.74(5.04-6.58)$ & 0.0139 & 0.2378 & $0.70(0.36-1.26)$ \\
\hline
\end{tabular}

181a-5p and miR-338-5p by Wilcoxon rank-sum test, respectively; table 3). Since extrahepatic metastasis is a component of the BCLC staging system, we eliminated the extrahepatic metastasis variable and conducted multivariate analysis using BCLC stage, DCP level, and the $\triangle \mathrm{Cq}$ of miR-181a-5p and miR-338-5p as covariables; only BCLC stage was revealed as an independent factor with borderline significance $[p=0.0475$, odds ratio (OR) of BCLC-C/BCLC-A for DC $0.14,95 \%$ confidence interval (CI) 0.02-0.94, and OR for BCLC-C/BCLC-B for DC 0.20, 95\% CI 0.04-0.93; table 3]. As sorafenib is usually recommended in BCLC-C cases, and miR181a-5p should be more suitable for quantification than miR-338-5p in a real clinical setting because of the lower interassay variation, we further conducted multivariate analysis using extrahepatic metastasis, serum DCP and miR-181a-5p levels before the treatment among 


\section{Cancer}

DOI: $10.1159 / 000449475$

(C) 2016 S. Karger AG, Basel www.karger.com/lic

Nishida et al.: MicroRNAs for the Prediction of Early Response to Sorafenib Treatment in Human Hepatocellular Carcinoma

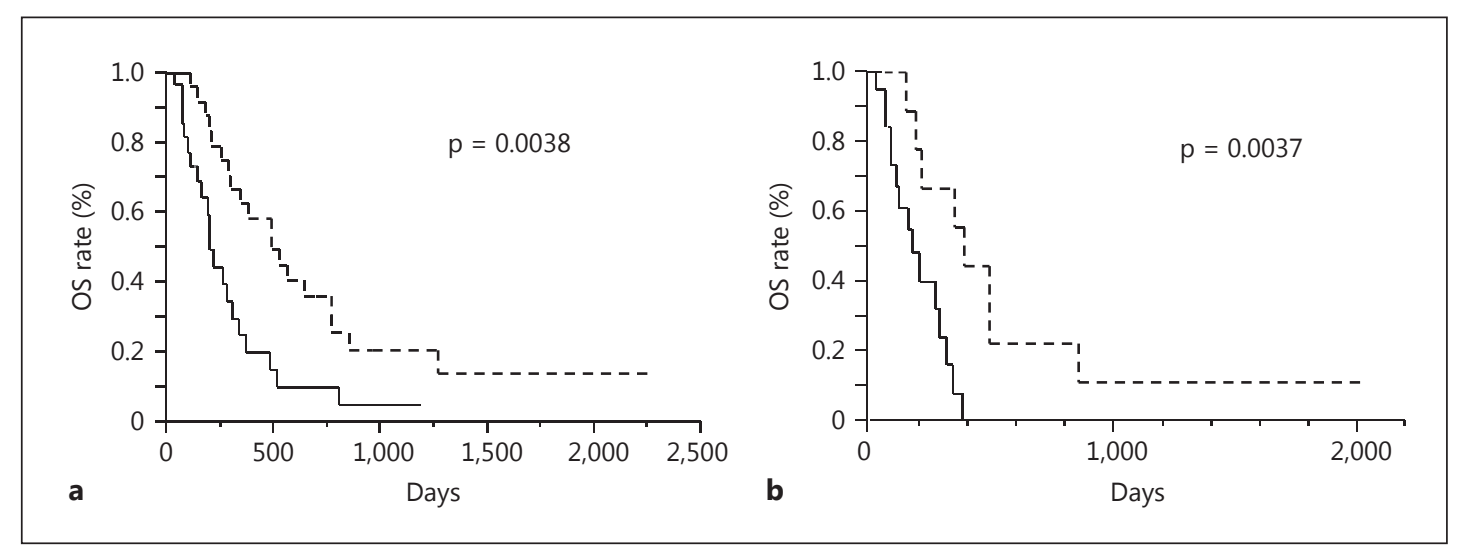

Fig. 4. Kaplan-Meier curve for OS after initiation of sorafenib treatment in serum miR-181a-5p high and low groups. The patients were categorized as the high serum miR-181a-5p and the low serum miR-181a-5p groups based on the $\Delta \mathrm{Cq}$ cutoff value of 3.25. The solid line shows the survival curve for the low serum miR181a-5p group and the dashed line for the high serum miR-181a-5p group. $p$ values by log-rank test are shown. a Kaplan-Meier curve for all HCC patients. Among the 53 patients examined, 25 were classified as the high serum miR-181a-5p group ( 6 were censored cases) and 28 were members of the low serum miR-181a$5 p$ group ( 7 were censored). Median periods of OS (25th-75th percentile) were 489 days (272-849) for the high serum miR-181a-5p group and 198 days (111-336) for the low serum miR-181a-5p group. b KaplanMeier curve among patients with BCLC-C HCC. Among the 30 HCC patients with BCLC-C tumor, 10 were classified as the high serum miR-181a-5p group ( 2 were censored cases) and 20 were members of the low serum miR-181a-5p group ( 5 were censored). Median periods of OS (25th-75th percentile) were 382 days (204489) for the high serum miR-181a-5p group and 164 days (80-279) for the low serum miR-181a-5p group.

BCLC-C cases. The analysis revealed that the $\Delta \mathrm{Cq}$ of miR-181a-5p, representing the serum miRNA levels, was the only independent factor for predicting DC $(\mathrm{p}=0.0092$, OR for DC $=$ $0.139,95 \%$ CI 0.011-0.658).

\section{Serum miR-181a-5p Level Predicts Survival after Sorafenib Treatment}

Finally, we determined the serum $\Delta \mathrm{Cq}$ of miR-181a-5p before treatment that best reflected the DC at 3 months after initiation of sorafenib using receiver operating characteristic analysis. A $\Delta$ Cq cutoff value of 3.25 provides the best discrimination of DC cases from non-DC cases with a sensitivity of 0.7500 and 1 - specificity of 0.3243 (online suppl. fig. 2).

Using the $\Delta$ Cq cutoff value of 3.25 , we classified the HCC patients into the high serum miR181a-5p and the low serum miR-181a-5 groups, and analyzed the survival after the initiation of sorafenib treatment using patient's death as an endpoint. The univariate analysis revealed that OS was significantly longer in patients with a high serum miR-181a-5p level than in those with a low level among all 53 HCC cases as well as the 30 BCLC-C cases ( $p=0.0038$ and 0.0037 for all the cases and the BCLC-C cases, respectively, fig. 4a, b). Among other clinical characteristics, such as age, sex, maximum tumor size, number of tumors, presence of extrahepatic metastasis and portal vein thrombosis, number of tumors was also significantly associated with the duration of OS among the BCLC-C cases ( $\mathrm{p}=0.0194$ by log-rank test, online suppl. table 2). We further conducted multivariate analysis using number of tumor (categorized as $\leq 5$ and $\geq 6$ ) and serum miR-181a-5p level as covariables and found that miR-181a-5p level was the independent factor for OS ( $\mathrm{p}=0.0194$, hazard ratio 0.267 , and $95 \% \mathrm{CI} 0.070-0.818$, online suppl. table 2). 


\section{Liver Cancer}

\section{Discussion}

Despite recent progress in the diagnosis and treatment of HCC, the prognosis of patients with advanced HCC is still unsatisfactory. Currently, sorafenib is the only systemic agent that shows improvement of progression-free and overall survival for advanced HCC patients with vascular invasion and extrahepatic spread, or in those who failed to respond to TACE $[2,3]$. Previously, we reported that DC after initiation of sorafenib is associated with improved OS in patients with advanced HCC [6]. Therefore, prediction of the initial response to this molecular-targeting agent is critical for the management of HCC, the needs of which are still unmet. In this study, we addressed this important issue and showed serum miRNA profiles that are associated with DC after the initiation of sorafenib treatment.

It is well described that miRNAs target several molecules that are critical for the development and biological behavior of cancer including HCC. So far, a number of studies have shown the role of miRNAs in the pathogenesis of liver disease including HCC [19]. Moreover, miRNAs detected in circulating blood are usually present within microvesicles such as exosomes [20, 21]; the circulating miRNAs could be markers of HCC progression [22]. However, it is difficult to evaluate the serum miRNA profiles from cancer cells for several reasons. For example, serum miRNA levels may be affected by the degradation of noncancerous cells, especially those derived from hematopoietic cells [14]. In addition, as serum miRNAs should be in exosomes as well as apoptotic bodies, a certain type of miRNA could be overestimated in HCC cases with degradation and vascular invasion [19]. Furthermore, serum miRNA levels are generally low, and the application of a universal internal control of miRNAs for quantification is controversial [14]. To address these issues, we used a qPCR panel specially focused on detecting serum miRNAs that have been previously reported in the literature. [18] For this PCR panel, we used LNA probes to provide enhanced sensitivity and specificity of qPCR with five reference miRNAs serving as an internal control. We also used ideal monitors for several steps of the quantification procedure. Through the analysis of screening and validation, we found miR-181a-5p is the best candidate as a serum biomarker for predicting early response of sorafenib in HCC treatment.

In this study, we applied the comparative cycle threshold methods for relative quantification for evaluating serum miRNAs [23], and found that an increase in $\triangle \mathrm{Cq}$ was detected in HCC patients with non-DC compared to DC patients for miR-181a-5p and miR-338-5p. As $\Delta C q$ was calculated as a difference of $\mathrm{Cq}$ between target and control miRNAs (Cq of target subtracted by that of control), a larger $\Delta \mathrm{Cq}$ implies a lower level of serum miRNAs. Interestingly, there is no statistical difference in the serum level of these miRNAs between HCC patients with DC and normal healthy controls; a significant decrease in serum levels was detected in HCC patients with non-DC for serum miR-181a-5p and miR-338-5p. In addition, a dose-dependent relationship was observed between serum miRNA levels and tumor responses, where the serum level was the highest in the PR and the lowest in the PD group, suggesting a biological role of serum miRNAs in tumor behavior; miR-181a-5p and miR338-5p in serum might provide a tumor-suppressive effect during sorafenib therapy. Currently, the role of miR-181a-5p and miR-338-5p in the pathogenesis of cancer is controversial. Previous reports suggest that the plasma level of miR-338-5p is higher in HCC patients than in healthy controls [24]. On the other hand, the expression of pre-miR-338-5p induced a decrease in cell proliferation and cell cycle arrest in glioblastoma cells; miR-338-5p sensitizes tumors to radiation through regulation of genes involved in DNA damage response [25]. Our result suggested that miR-338-5p might have a role in the induction of tumor response to sorafenib treatment, although the quantification of serum miR-338-5p is unstable compared to that of miR-181a-5p because of a lower level of the initial copy number. Regarding miR$181 a-5 p$, the overexpression in tumorous tissue was reportedly associated with shorter 
survival in patients with ovarian cancer who underwent neoadjuvant chemotherapy [26]. However, another report suggested that a reduction of serum miR-181a-5p was observed in breast cancer patients [27]. Recently, it was reported that miR-181a-5p inhibits cancer cell migration and angiogenesis through downregulation of matrix metalloproteinase-14 in aggressive human breast and colon cancers [28]. More importantly, it has also been reported that miR-181a-5p, which directly targets c-Met, is downregulated in HCC and suppresses motility, invasion, and branching-morphogenesis; the loss of miR-181a-5p expression led to the activation of c-Met-mediated oncogenic signaling in hepatocarcinogenesis [29]. A recent study suggested that c-Met could activate p38 $\alpha$ mitogen-activated protein kinase, which could be a molecule involved in the escape pathway of sorafenib-resistance [30]. Taken together, it is conceivable that miR-181a-5p acts as a tumor suppressor in HCC, and its expression might have a synergic antitumor effect with the multikinase inhibitor. Further study is required to understand the role of miR-181a-5p in HCC pathogenesis and chemoresistance.

As mentioned before, we have reported that DC after the initiation of sorafenib is associated with improved OS in patients with advanced HCC [6]; the presented data also show that high serum miR-181a-5p levels are associated with both high DCR and long OS in patients with BCLC-C HCC, although the data might be biased because of the nature of retrospective analyses. Therefore, analyzing a miR-181a-5p level should be informative for initiating sorafenib treatment in HCC with advanced stage.

Although we screened candidate miRNAs for predicting DC in sorafenib treatment using a serum secreted miRNA panel and confirmed the relationships with validation cohort, the limitation of this study is mainly attributed to the relatively small size of the sample. Therefore, the results of this study should be reevaluated using a larger cohort. Further study will allow us to understand the predictive value of serum miRNAs in the tumor behavior during sorafenib therapy.

\section{Acknowledgements}

The authors thank Ms. Ikuko Kagami for technical assistance. This work was supported in part by a grant-in-aid for scientific research (KAKENHI: 15K09028) from the Japanese Society for the Promotion of Science.

\section{Disclosure Statement}

The authors declare that they have no conflict of interest.

\section{References}

1 Torre LA, Bray F, Siegel RL, Ferlay J, Lortet-Tieulent J, Jemal A: Global cancer statistics, 2012. CA Cancer J Clin 2015;65:87-108.

2 Kudo M: Treatment of advanced hepatocellular carcinoma with emphasis on hepatic arterial infusion chemotherapy and molecular targeted therapy. Liver Cancer 2012;1:62-70.

3 Llovet JM, Ricci S, Mazzaferro V, Hilgard P, Gane E, Blanc JF, de Oliveira AC, Santoro A, Raoul JL, Forner A, Schwartz M, Porta C, Zeuzem S, Bolondi L, Greten TF, Galle PR, Seitz JF, Borbath I, Haussinger D, Giannaris T, Shan M, Moscovici M, Voliotis D, Bruix J; SHARP Investigators Study Group: Sorafenib in advanced hepatocellular carcinoma. N Engl J Med 2008;359:378-390.

4 Bruix J, Sherman M, American Association for the Study of Liver Diseases: Management of hepatocellular carcinoma: an update. Hepatology 2011;53:1020-1022. 
5 Yamasaki T, Kimura T, Kurokawa F, Aoyama K, Ishikawa T, Tajima K, Yokoyama Y, Takami T, Omori K, Kawaguchi K, Tsuchiya M, Terai S, Sakaida I, Okita K: Prognostic factors in patients with advanced hepatocellular carcinoma receiving hepatic arterial infusion chemotherapy. J Gastroenterol 2005;40:70-78.

6 Arizumi T, Ueshima K, Chishina H, Kono M, Takita M, Kitai S, Inoue T, Yada N, Hagiwara S, Minami Y, Sakurai T, Nishida N, Kudo M: Duration of stable disease is associated with overall survival in patients with advanced hepatocellular carcinoma treated with sorafenib. Dig Dis 2014;32:705-710.

7 Borel F, Konstantinova P, Jansen PL: Diagnostic and therapeutic potential of miRNA signatures in patients with hepatocellular carcinoma. J Hepatol 2012;56:1371-1383.

8 Bai S, Nasser MW, Wang B, Hsu SH, Datta J, Kutay H, Yadav A, Nuovo G, Kumar P, Ghoshal K: MicroRNA-122 inhibits tumorigenic properties of hepatocellular carcinoma cells and sensitizes these cells to sorafenib. J Biol Chem 2009;284:32015-32027.

9 Shimizu S, Takehara T, Hikita H, Kodama T, Miyagi T, Hosui A, Tatsumi T, Ishida H, Noda T, Nagano H, Doki Y, Mori M, Hayashi N: The let-7 family of microRNA inhibits Bcl-xL expression and potentiates sorafenib-induced apoptosis in human hepatocellular carcinoma. J Hepatol 2010;52:698-704.

10 Mao K, Zhang J, He C, Xu K, Liu J, Sun J, Wu G, Tan C, Zeng Y, Wang J, Xiao Z: Restoration of miR-193b sensitizes hepatitis B virus-associated hepatocellular carcinoma to sorafenib. Cancer Lett 2014;352:245-252.

11 Gyongyosi B, Vegh E, Jaray B, Szekely E, Fassan M, Bodoky G, Schaff Z, Kiss A: Pretreatment microRNA level and outcome in sorafenib-treated hepatocellular carcinoma. J Histochem Cytochem 2014;62:547-555.

12 Vaira V, Roncalli M, Carnaghi C, Faversani A, Maggioni M, Augello C, Rimassa L, Pressiani T, Spagnuolo G, Di Tommaso L, Fagiuoli S, Rota Caremoli E, Barberis M, Labianca R, Santoro A, Bosari S: MicroRNA-425-3p predicts response to sorafenib therapy in patients with hepatocellular carcinoma. Liver Int 2015;35:1077-1086.

13 Nishida N, Kitano M, Sakurai T, Kudo M: Molecular mechanism and prediction of sorafenib chemoresistance in human hepatocellular carcinoma. Dig Dis 2015;33:771-779.

14 Ono S, Lam S, Nagahara M, Hoon DS: Circulating microRNA biomarkers as liquid biopsy for cancer patients: pros and cons of current assays. J Clin Med 2015;4:1890-1907.

15 Lind K, Stahlberg A, Zoric N, Kubista M: Combining sequence-specific probes and DNA binding dyes in realtime PCR for specific nucleic acid quantification and melting curve analysis. BioTechniques 2006;40:315-319.

16 Lencioni R, Llovet JM: Modified RECIST (mRECIST) assessment for hepatocellular carcinoma. Semin Liver Dis 2010;30:52-60.

17 Gadkar V, Filion M: New developments in quantitative real-time polymerase chain reaction technology. Curr Issues Mol Biol 2014;16:1-6.

18 Ameling S, Kacprowski T, Chilukoti RK, Malsch C, Liebscher V, Suhre K, Pietzner M, Friedrich N, Homuth G, Hammer E, Volker U: Associations of circulating plasma microRNAs with age, body mass index and sex in a population-based study. BMC Med Genomics 2015;8:61.

19 Szabo G, Bala S: MicroRNAs in liver disease. Nat Rev Gastroenterol Hepatol 2013;10:542-552.

20 Reid G, Kirschner MB, van Zandwijk N: Circulating microRNAs: association with disease and potential use as biomarkers. Crit Rev Oncol Hematol 2011;80:193-208.

21 Lemoinne S, Thabut D, Housset C, Moreau R, Valla D, Boulanger CM, Rautou PE: The emerging roles of microvesicles in liver diseases. Nat Revi Gastroenterol Hepatol 2014;11:350-361.

22 Murakami Y, Toyoda H, Tanahashi T, Tanaka J, Kumada T, Yoshioka Y, Kosaka N, Ochiya T, Taguchi YH: Comprehensive miRNA expression analysis in peripheral blood can diagnose liver disease. PLoS One 2012;7:e48366.

23 Goll R, Olsen T, Cui G, Florholmen J: Evaluation of absolute quantitation by nonlinear regression in probebased real-time PCR. BMC Bioinformatics 2006; 7:107.

24 Chen Y, Chen J, Liu Y, Li S, Huang P: Plasma miR-15b-5p, miR-338-5p, and miR-764 as biomarkers for hepatocellular carcinoma. Med Sci Monit 2015;21:1864-1871.

25 Besse A, Sana J, Lakomy R, Kren L, Fadrus P, Smrcka M, Hermanova M, Jancalek R, Reguli S, Lipina R, Svoboda M, Slampa P, Slaby O: MiR-338-5p sensitizes glioblastoma cells to radiation through regulation of genes involved in DNA damage response. Tumour Biol 2016;37:7719-7727.

26 Petrillo M, Zannoni GF, Beltrame L, Martinelli E, DiFeo A, Paracchini L, Craparotta I, Mannarino L, Vizzielli G, Scambia G, D'Incalci M, Romualdi C, Marchini S: Identification of high-grade serous ovarian cancer miRNA species associated with survival and drug response in patients receiving neoadjuvant chemotherapy: a retrospective longitudinal analysis using matched tumor biopsies. Ann Oncol 2016;27:625-634.

27 Ferracin M, Lupini L, Salamon I, Saccenti E, Zanzi MV, Rocchi A, Da Ros L, Zagatti B, Musa G, Bassi C, Mangolini A, Cavallesco G, Frassoldati A, Volpato S, Carcoforo P, Hollingsworth AB, Negrini M: Absolute quantification of cell-free microRNAs in cancer patients. Oncotarget 2015;6:14545-14555.

28 Li Y, Kuscu C, Banach A, Zhang Q, Pulkoski-Gross A, Kim D, Liu J, Roth E, Li E, Shroyer KR, Denoya PI, Zhu X, Chen L, Cao J: MiR-181a-5p inhibits cancer cell migration and angiogenesis via downregulation of matrix metalloproteinase-14. Cancer Res 2015;75:2674-2685.

29 Korhan P, Erdal E, Atabey N: Mir-181a-5p is downregulated in hepatocellular carcinoma and suppresses motility, invasion and branching-morphogenesis by directly targeting c-Met. Biochem Biophys Res Commun 2014;450:1304-1312.

30 Rudalska R, Dauch D, Longerich T, McJunkin K, Wuestefeld T, Kang TW, Hohmeyer A, Pesic M, Leibold J, von Thun A, Schirmacher P, Zuber J, Weiss KH, Powers S, Malek NP, Eilers M, Sipos B, Lowe SW, Geffers R, Laufer S, Zender L: In vivo RNAi screening identifies a mechanism of sorafenib resistance in liver cancer. Nat Med 2014;20:1138-1146. 